\title{
Cluster A Personality Symptomatology in Youth
}

\author{
Thomas A. Widiger
}

Published online: 16 October 2010

(C) Springer Science+Business Media, LLC 2010

\begin{abstract}
There is an increasing recognition of the importance of studying the childhood antecedents of adult personality disorders as well as personality disorders themselves within childhood and adolescence. This has been a long neglected area of research. The two papers within this special issue of Journal of Psychopathology and Behavioral Assessment by Esterberg et al. (2010) and De Clercq et al. (2010) make significant strides toward addressing this delinquent focus of investigation. This commentary addresses three issues stimulated by these two particular studies: the distinction between personality and other mental disorders, dimensional models of classification, and openness to experience.
\end{abstract}

Keywords Personality disorder - Cluster A - Children . Adolescents

\section{Introduction}

The American Psychiatric Association's (APA) personality disorders section of the Diagnostic and Statistical Manual of Mental Disorders (DSM-IV-TR; APA, 2000) is organized into three clusters. "Cluster A" includes the paranoid, schizoid, and schizotypal personality disorders. Esterberg et al. (2010) and De Clercq et al. (2010) provide very informative findings and discussions of the consideration of Cluster A personality disorder symptomatology within children and adolescents. This commentary is organized

\section{T. A. Widiger $(\bowtie)$}

Department of Psychology, University of Kentucky,

115 Kastle Hall,

Lexington, KY 40506-0044, USA

e-mail: widiger@uky.edu with respect to three main concerns that follow from these incisive papers: (1) the differentiation of personality from other mental disorders, (2) dimensional classification of Cluster A symptomatology, and (3) openness to experience. Each will be discussed in turn.

\section{Differentiation of Personality from Other Mental Disorders}

Personality disorders are currently diagnosed on a separate axis from most other mental disorders in DSM-IV-TR (APA 2000). Most of the mental disorders are placed on Axis I, whereas personality disorders (and mental retardation) are placed on Axis II. This separate axis placement implemented in DSM-III (APA 1980) stimulated a substantial increase in the recognition of personality disorders by clinicians and probably as well by researchers (Widiger 2003). Prior to DSM-III personality disorders were often neglected as clinicians' primary attention and focus was on an Axis I condition, such as a mood, anxiety, or psychotic disorder. Clinicians tended to consider personality and other mental disorders as being mutually exclusive. They felt that both should not, and perhaps could not, be diagnosed within the same patient. This misunderstanding was addressed well by their placement on separate axes (Spitzer et al. 1980). Once a clinician has provided an Axis I diagnosis, his or her attention is then shifted to Axis II, wherein the possible presence of a co-occurring personality disorder diagnosis is then considered. The separate axis placement has also been useful in recognizing that every patient has a personality, and many will have personality disorders. The respective personality disorders (and personality more generally) can have an important effect upon the treatment and course of many Axis I disorders, as well as etiology. 
The multiaxial system introduced in DSM-III is likely to be abandoned in DSM-5. The rationale for its removal has not been explained by the authors of DSM-5 (see www. DSM5.org for the rationale for other proposed changes). One potential reason is that there is no consistent distinction between a personality disorder and an Axis I mental disorder (Krueger 2005; Widiger 2003). In addition, the separate axis placement has contributed to a misperception that personality disorders are not treatable and therefore should not receive financial (insurance) coverage (Widiger 2003). Neither of these two concerns, however, address the considerable benefits within clinical practice and research obtained by their separate placement. Shifting personality disorders to Axis I is likely to result in their loss of recognition by clinicians, and may as well have a comparable impact on the attention of researchers.

There are two different ways of understanding a shift of personality disorders, such as schizotypal, to Axis I. One possibility is that personality disorders will continue to occupy a separate, distinct section of the diagnostic manual, albeit this section is within Axis I. The other possibility is that the concept of a personality disorder will be abandoned entirely and each of the currently existing personality disorders will be folded into an Axis I disorder as an early onset, chronic variant of that respective condition (e.g., avoidant personality disorder would become generalized social phobia).

The latter proposal might seem unlikely to many clinicians and researchers, but it was in fact an explicit proposal raised during the first DSM-5 research planning conference, a summary of which was provided by First et al. (2002). Nor is such a proposal in fact at all unusual for DSM-IV-TR schizotypal personality disorder (SPD) because this particular personality disorder is already classified within the World Health Organization's (WHO) International Classification of Mental Disroders (WHO 1992, see p. 95) as a form of schizophrenia (i.e., schizotypal disorder) rather than as a personality disorder.

It is the position of this commentary, however, that it will be important, both scientifically and clinically, to continue to recognize the existence of personality disorders within the APA diagnostic manual as disorders distinct from other conditions (Widiger 2003), as it is important to recognize the existence of personality. However, a concern common to both Esterberg et al. (2010) and De Clercq et al. (2010) is that this distinction is at times rather fluid, if not lost entirely.

For example, Esterberg et al. stated that "based on studies of the prodrome [phase of schizophrenia] it is estimated that between $25-45 \%$ of those diagnosed with SPD in adolescence go on to develop schizophrenia." However, research concerning the prodromal phase of schizophrenia may not be relevant to an understanding of
SPD. The prodromal phase of schizophrenia is not SPD. If a distinction is to be made between SPD as a personality disorder, and schizophrenia as an Axis I disorder, it would seem important to distinguish between the prodomal phase of schizophrenia and SPD.

It is true that the prodromal phase of schizophrenia and SPD are very similar in content. A proposal for DSM-III-R was to in fact make them identical (Widiger et al. 1988). This proposal was rejected though due to the fact that there are some notable difference in content (e.g., prodromal phase can include fatigue and lack of interest) and making them equivalent would be inconsistent with the primary function of the manual to facilitate differential diagnosis.

In DSM-IV-TR (APA 2000) it is stated that "schizotypal personality disorder should not be diagnosed if the pattern of behavior occurs exclusively during the course of schizophrenia" (p. 698). It is acknowledged that the prodromal phase of schizophrenia and SPD are quite similar to one another and both may be first evident during adolescence, but if what had been originally diagnosed as SPD is followed by an active phase of schizophrenia, then most likely the correct diagnosis during adolescence would have been the prodromal phase of schizophrenia rather than SPD. Once the active phase of schizophrenia is evident, one can still provide, for the historical record, a diagnosis of SPD but it would carry the further designation of being "premorbid" rather than comorbid (APA 2000). If "schizotypal" symptoms continue after the remission of the active phase the correct diagnosis would be schizophrenia, residual phase, rather than a return of SPD.

Esterberg et al. (2010) also stated that "for many patients, the prodromal phase of Axis I psychotic disorders, such as schizophrenia, begins with SPD in adolescence." Perhaps it should be said instead that these purported cases of SPD in adolescence were more accurately diagnosed, in hindsight, as having been an extended prodromal phase of schizophrenia. Personality disorders and Axis I disorders can be associated with one another in a variety of ways (Widiger and Smith 2008), including an etiological relationship (one disorder contributes to the etiology of the other), a pathoplastic relationship (one disorder altering the course or presentation of another), and a spectrum relationship (the two disorders are really one disorder sharing a common etiology and pathology). SPD, as a prodromal phase of schizophrenia, is perhaps best understood within a spectrum relationship, wherein SPD and schizophrenia are not separate, distinct disorders present at two different points in time, but are instead one, single disorder being mistakenly provided with two different diagnoses.

A similar conclusion can be reached with respect to some of the findings from the Esterberg et al. longitudinal study. They indicated that a small number of their 36 cases 
of SPD diagnosed within adolescence met criteria for schizophrenia $(n=3)$ or schizoaffective $(n=2)$ disorder at one-year follow-up. As they suggested, these cases "converted to psychosis" and would no longer be considered cases of SPD. It is perhaps not so much that the disorder has changed over time, but that it was (understandably) misdiagnosed at the onset of the study because the entire course of the schizophrenia or schizoaffective disorder had not yet become apparent. Only the cases diagnosed with SPD that continue to meet criteria for SPD at one-year follow-up are more confidently and accurately considered to be true cases of SPD.

Related concerns can be raised with respect to De Clercq et al. (2010). They studied 194 boys with an autistic, Asperger's or other pervasive developmental disorder (i.e., autism spectrum disorders or ASD). If these developmental disorders were conceptualized as personality disorders, they would likely be most comfortably included within Cluster A. De Clercq et al. correctly point out that there has been considerable difficulty developing a reliable and valid classification of this spectrum of developmental psychopathology and they suggest that some clarity might be derived through considering these conditions from the perspective of a dimensional model of maladaptive personality structure, such as the Dimensional Personality Symptom Itempool (DIPSI; De Clercq et al. 2006). They found significant relationships of maladaptive DIPSI traits with ASD symptoms concerning restricted interests and repetitive behaviors but not so much with the autism symptoms involving communication and language deficits. They suggest that the former may be best understood as maladaptive variants of general personality "and the extent to which common factors for these constructs exist, etiologic investigations may be most fruitful when examining their joint relationships." More specifically, the "personality traits may provide important insights into future research on causal factors implicated in the development of ASD symptoms."

De Clercq et al. (2010) may indeed be correct that much can be learned about ASD when at least some aspect of its features are understood from the perspective of more general personality functioning. There seems to be little doubt that some of the symptoms and features of ASD shade into general personality traits. Nigg and colleagues have similarly demonstrated the value of considering both child and adult features of attention-deficit hyperactivity disorder (ADHD) in terms of the five-factor model (FFM) of general personality structure (Martel et al. 2010; Nigg et al. 2002). ADHD is considered by many to be grossly under-recognized within adulthood due in part to difficulties in understanding how it is expressed after childhood and adolescence. Nigg and colleagues suggest that it may be best understood as (primarily) a variant of low FFM conscientiousness, comparable to how other childhood disruptive behavior disorders (i.e., conduct and oppositional defiant disorder) have relatively clear personality correlates when they continue into adulthood.

However, it is also possible that the similarities between ASD and the personality traits assessed by the DIPSI may prove to be only superficially similar rather than represent a true spectrum relationship. Persons diagnosed with schizophrenia will evidence elevations on (for instance) FFM introversion, as well as other domains of the FFM, yet one would not actually suggest that schizophrenia is a disorder of personality or that there is a common etiology for schizophrenia and FFM introversion. The association in this instance might be more pathoplastic than spectrum, wherein the Axis I pathology of schizophrenia causes a change to the appearance of personality (Widiger 2009; Widiger and Smith 2008). The mood disorder of depression similarly causes persons to describe themselves as being elevated on personality measures of neuroticism, but such apparent yet temporary shifts in the appearance of personality are not generally understood to represent true changes in personality functioning (Widiger 2009; albeit see Costa et al. 2005, for a different opinion). Lending some support for this concern is that the personality correlates of ASD in De Clercq et al. were not terribly specific to a particular domain of the DIPSI.

\section{Dimensional Classification of Cluster A Symptomatology}

A notable strength of De Clercq et al. (2010) relative to Esterberg et al. (2010) is their application of a dimensional model of general personality structure rather than relying on the faulty and faltering DSM-IV-TR personality disorder diagnostic categories. Esterberg et al. provide follow-up data for persons diagnosed with DSM-IV-TR schizoid personality disorder (SZPD), paranoid personality disorder (PPD), and SPD. It appears that PPD and SPD will not be included in the next edition of the diagnostic manual (Skodol 2010). It is no coincidence that Esterberg et al. indicate that research on the childhood antecedents of SZPD and PPD is sorely lacking, relative to SPD. One might then wonder though whether it is now even useful to report findings for diagnoses that will no longer be recognized. Few persons still report findings for the passive-aggressive, aesthenic, and inadequate personality disorders, diagnoses deleted from earlier editions of the manual.

An additional difficulty with the DSM-IV-TR diagnostic categories has been their diagnostic co-occurrence and instability (Widiger and Trull 2007), a problem that is again apparent in the findings of Esterberg et al. They report that at the time of the initial assessments $36 \%$ and $27 \%$ of those 
diagnosed with SPD also met criteria for SZPD and PPD, respectively. Even more problematic was that $34 \%$ of the initial SPD cases no longer met criteria for SPD at one-year follow-up but did meet criteria for another personality disorder, predominately either SZPD or PPD.

Shifts in personality disorder diagnoses over time has not been heavily researched but are clearly problematic for their validity. Would anyone really argue that persons' personality disorders are shifting over time, changing from SPD to PPD in just 1 year? Fundamental to the concept of personality, and personality disorder, is temporal stability. This does not necessarily imply that no change in personality will occur, particularly for adolescents. Even normal personality functioning does change over time. But, shifts from one personality disorder diagnosis to another within 1 year does raise questions as to the validity of the initial diagnosis, the follow-up diagnosis, or even both. The shift in diagnoses reported by Esterberg et al. are most likely due to the overlap in symptomatology among the diagnostic categories and the arbitrary diagnostic thresholds, rather than true changes in personality functioning.

The participants in Esterberg et al. (2010) probably had complex constellations of maladaptive personality traits, including some (but not all) of the traits of SPD, SZPD, and PPD. Some of the participants were perhaps just below threshold for one or more of the three diagnostic categories at the onset of the study, whereas other participants were probably just above threshold. Due to the less than perfect reliability (and validity) of personality disorder assessment, minor shifts in the assessment will occur over time, resulting in major shifts in the categorical diagnoses.

A dimensional classification of personality disorder, as provided, for instance, by the DIPSI (De Clercq et al. 2006), will provide a more reliable as well as more precise description of each youth's maladaptive personality trait profile. Rather than lump the participants into overlapping categories that are defined and distinguished by arbitrary thresholds, the DIPSI provides a more precise description of each individual's relatively unique maladaptive personality trait profile. The constructs assessed by the DIPSI are also more homogeneous and distinct than those assessed by Esterberg et al. Problematic diagnostic co-occurrence and radical shifts in personality description are then much less likely to occur. Research has indeed indicated better temporal stability for these dimensional trait profiles than is typically obtained for the diagnostic categories (Warner et al. 2004; Widiger and Trull 2007).

\section{Openness to Experience}

A potential disadvantage, however, of the DIPSI, particularly when used to assess DSM-IV-TR Cluster A symp- tomatology, is that it does not include much depth in the assessment of a fifth domain of personality, openness to experience. The DIPSI was constructed to assess the maladaptive variants of the personality traits of children and adolescents assessed by the Hierarchical Personality Inventory for Children (HiPIC; Mervielde and De Fruyt 2002). The HiPIC assesses five broad domains of personality functioning, including conscientiousness, benevolence, extraversion, imagination, and emotional stability. These are aligned conceptually and empirically with the adult FFM domains of conscientiousness, agreeableness, extraversion, openness, and neuroticism, respectively (Mervielde and De Fruyt 2002).

The DIPSI, however, does not include the HiPIC imagination or the FFM openness to experience domains of personality functioning, due largely to finding that there was an insufficient prevalence of the maladaptive variants of these traits within children and adolescents to warrant their inclusion as one of the broad domains (De Clercq et al. 2006). This findings is consistent with adult personality disorder research in which the maladaptive variants of FFM openness to experience do not occur with sufficient prevalence to carry a fifth factor of maladaptive personality (O'Connor 2005; Saulsman and Page 2004; Widiger and Simonsen 2005).

However, Tackett et al. (2008) demonstrated empirically that a fifth factor of personality disorder symptomatology (which they identified as "peculiarity") does emerge when a sufficient representation of cognitiveperceptual aberrations are included. An additional study has suggested that this fifth factor would not align with FFM openness or with HiPIC imagination, finding instead that two separate factors emerge, one concerning normal openness to experience and the other concerning maladaptive traits of peculiarity (Watson et al. 2008). This unique finding is a bit surprising, as it suggests that four of the domains of general personality have both normal and abnormal variants but one domain (openness to experience or imagination) has no maladaptive variants and a sixth domain (peculiarity) has no normal variants. This particular finding though is readily understood as a complementary artifact of overloading a particular domain (Samuel and Widiger 2008). Just as a domain of personality functioning will not appear if it does not have adequate representation among the variables submitted to a factor analysis, a domain of personality functioning will likely split if its representation is excessive, relative to the others.

There is a considerable body of research that does suggest that the maladaptive variants of FFM openness to experience and HiPIC imagination would be the cognitiveperceptual aberrations often seen in persons with DSM-IV Cluster A symptomatology (Ross et al. 2002). For example, 
in other factor analytic studies by Camisa et al. (2005), Kwapil et al. (2008) and Wiggins and Pincus (1989) cognitive-perceptual aberrations clearly load on the FFM openness factor. SPD researchers (Lynam and Widiger 2001) and practicing clinicians (Samuel and Widiger 2004) both describe prototypic cases of SPD as involving high levels of openness to experience.

The relationship of openness to experience to DSM-IVTR Cluster A symptomatology is embedded explicitly in a number of adult measures of openness. For example, another measure of this domain is provided by the HEXACO-Personality Inventory (HEXACO-PI; Lee and Ashton 2004). The HEXACO-PI Openness to Experience scale corresponds conceptually and empirically with FFM openness. This HEXACO-PI scale includes four facet scales, one of which is titled Unconventionality that assesses the disposition to be eccentric, weird, peculiar, odd, and strange. Piedmont et al. (2009) developed scales to assess maladaptive variants of high and low FFM openness. The "Odd and Eccentric" openness scale correlates substantially with schizotypal personality disorder and paranormal beliefs. Another alternative assessment of this domain is provided by the Unconventionality scale within the Big Seven model of Tellegen (Tellegen and Waller 1987). Big Seven unconventionality is aligned explicitly with FFM openness and includes a number of items that resemble closely normal openness (e.g., curious, inquisitive, imaginative, and creative) as well as items that concern such attributes as dwelling upon fantasies, having ideas or beliefs that have little basis within reality, or often engaging in activities that are bizarre, deviant, or aberrant (Tellegen and Waller 1987). Van Kampen (2009) includes a comparable scale within his 5-Dimensional Personality Test (5DPT). The 5-DPT assesses the domains of neuroticism, extraversion, absorption, insensitivity, and orderliness. 5DPT absorption aligns explicitly with FFM openness and assesses dissociative absorption, positive symptoms of schizotypy, and other comparable maladaptive variants (Van Kampen 2009).

In sum, a fully comprehensive dimensional model of normal and abnormal personality functioning would include a fifth domain of personality, assessing the normal variant of openness to experience (or, in childhood, imagination), along with its maladaptive variants of cognitive-perceptual aberrations. This domain was understandably not included within the DIPSI, given that this symptamotology is not as prevalent as the maladaptive variants of HiPIC conscientiousness, benevolence, extraversion, and emotional stability. Nevertheless, in some particular contexts, such as studies concerning Cluster A symptomatology in children and adolescents, maladaptive variants of childhood imagination and adult openness to experience will likely need greater breadth and depth of assessment.

\section{Conclusions}

There is an increasing recognition of the importance of studying the childhood antecedents of adult personality disorders as well as personality disorders within childhood and adolescence (Cicchetti and Crick 2009; Tackett et al. 2009). This has been a long neglected area of research. The two papers within this special issue of Journal of Psychopathology and Behavioral Assessment by Esterberg et al. (2010) and De Clercq et al. (2010) provide significant strides toward addressing this delinquent focus of investigation. This commentary addressed three issues stimulated by these two particular studies: the distinction between personality and other mental disorders, dimensional models of classification, and openness to experience. It is hoped that these two studies and this commentary will further stimulate continued research on childhood and adolescent personality disorder.

\section{References}

American Psychiatric Association. (1980). Diagnostic and statistical manual of mental disorders (3rd ed.). Washington: Author.

American Psychiatric Association. (2000). Diagnostic and statistical manual of mental disorders. Text Revision (4th ed.). Washington: Author.

Camisa, K. M., Bockbrader, M. A., Lysaker, P., Rae, L. L., Brenner, C. A., \& O'Donnell, B. F. (2005). Personality traits in schizophrenia and related personality disorders. Psychiatry Research, 133, 23-33.

Cicchetti, D., \& Crick, N. R. (2009). Precursors and diverse pathways to personality disorder in children and adolescents. Development and Psychopathology, 21, 683-685.

Costa, P. T., Bagby, R. M., Herbst, J. F., \& McCrae, R. R. (2005). Personality self-reports are concurrently reliable and valid during acute depressive episodes. Journal of Affective Disorders, 89, 45-55.

De Clercq, B., De Fruyt, F., Van Leeuwen, K., \& Mervielde, I. (2006). The structure of maladaptive personality traits in childhood: a first step toward an integrative developmental perspective for DSM-V. Journal of Abnormal Psychology, 115, 639-657.

De Clercq, B., Aelterman, N., De Pauw, S., De Bolle, M., Decuyper, M., \& Tackett, J. (2010). Delineating childhood autism spectrum symptoms From a maladaptive trait perspective. Journal of Psychopathology and Behavioral Assessment. doi:10.1007/ s10862-010-9191-8.

Esterberg, M. L., Goulding, S. M., \& Walker, E. F. (2010). Cluster A personality disorders: schizotypal, schizoid, and paranoid personality disorders in childhood and adolescence. Journal of Psychopathology and Behavioral Assessment. doi:10.1007/ s10862-010-9183-8.

First, M. B., Bell, C. B., Cuthbert, B., Krystal, J. H., Malison, R., Offord, D. R., et al. (2002). Personality disorders and relational disorders: A research agenda for addressing crucial gaps in DSM. In D. J. Kupfer, M. B. First, \& D. A. Regier (Eds.), A research agenda for $D S M-V$ (pp. 123-199). Washington: American Psychiatric Association.

Krueger, R. F. (2005). Continuity of Axes I and II: toward a unified model of personality, personality disorders, and clinical disorders. Journal of Personality Disorders, 19, 233-261. 
Kwapil, T. R., Barrantes-Vidal, N., \& Silvia, P. J. (2008). The dimensional structure of the Wisconsin Schizotypy Scales: factor identification and construct validity. Schizophrenia Bulletin, 34, 444-457.

Lee, K., \& Ashton, M. C. (2004). Psychometric properties of the HEXACO Personality Inventory. Multivariate Behavioral Research, 39, 329-358.

Lynam, D. R., \& Widiger, T. A. (2001). Using the five factor model to represent the DSM-IV personality disorders: an expert consensus approach. Journal of Abnormal Psychology, 110, 401-412.

Martel, M. M., Goth-Owens, T., Martinez-Torteya, C., \& Nigg, J. T. (2010). A person-centered personality approach to heterogeneity in attention-deficit/hyperactivity disorder (ADHD). Journal of Abnormal Psychology, 119, 186-196.

Mervielde, I., \& De Fruyt, F. (2002). Assessing children's traits with the Hierarchical Personality Inventory for Children. In B. De Raad \& M. Perugini (Eds.), Big five assessment (pp. 129-146). Seattle: Hogrefe \& Huber Publishers.

Nigg, J. T., John, O. P., Blaskey, L. G., Huang-Pollock, C. L., Willcutt, E. G., Hinshaw, S. P., et al. (2002). Big Five dimensions and ADHD symptoms: links between personality traits and clinical symptoms. Journal of Personality and Social Psychology, 83, 451-469.

O'Connor, B. P. (2005). A search for consensus on the dimensional structure of personality disorders. Journal of Clinical Psychology, 61, 323-345.

Piedmont, R. L., Sherman, M. F., Sherman, N. C., Dy-Liacco, G. S., \& Williams, J. E. (2009). Using the five-factor model to identify a new personality disorder domain: the case for experiential permeability. Journal of Personality and Social Psychology, 96, $1245-1258$.

Ross, S. R., Lutz, C. J., \& Bailey, S. E. (2002). Positive and negative symptoms of schizotypy and the five-factor model: a domain and facet level analysis. Journal of Personality Assessment, 79, 53-72.

Samuel, D. B., \& Widiger, T. A. (2004). Clinicians' descriptions of prototypic personality disorders. Journal of Personality Disorders, 18, 286-308.

Samuel, D. B., \& Widiger, T. A. (2008). A meta-analytic review of the relationships between the five-factor model and DSM-IV-TR personality disorders: a facet level analysis. Clinical Psychology Review, 28, 1326-1342.

Saulsman, L. M., \& Page, A. C. (2004). The five-factor model and personality disorder empirical literature: a meta-analytic review. Clinical Psychology Review, 23, 1055-1085.

Skodol, A. (2010). Rationale for proposing five specific personality types. Retrieved from http://www.dsm5.org/ProposedRevisions/ Pages/RationaleforProposingFiveSpecificPersonalityDisorder Types.aspx

Spitzer, R. L., Williams, J. B. W., \& Skodol, A. E. (1980). DSM-III: the major achievements and an overview. American Journal of Psychiatry, 137, 151-164.
Tackett, J. L., Silberschmidt, A. L., Krueger, R. F., \& Sponheim, S. R. (2008). A dimensional model of personality disorder: Incorporating DSM cluster A characteristics. Journal of Abnormal Psychology, 117, 454-459.

Tackett, J. L., Balsis, S., Oltmanns, T. F., \& Krueger, R. F. (2009). A unifying perspective on personality pathology across the life span: developmental considerations for the fifth edition of the Diagnostic and Statistical Manual of Mental Disorders. Development and Psychopathology, 21, 687-713.

Tellegen, A., \& Waller, N. G. (1987). Exploring personality through test construction: Development of the multidimensional personality questionnaire. Unpublished manuscript, Minneapolis, Minnesota.

Van Kampen, D. (2009). Personality and psychopathology: a theorybased revision of Eyenck's PEN model. Clinical Practice and Epidemiology in Mental Health, 5, 9-21.

Warner, M. B., Morey, L. C., Finch, J. F., Gunderson, J. G., Skodol, A. E., Sanislow, C. A., et al. (2004). The longitudinal relationship of personality traits and disorders. Journal of Abnormal Psychology, 113, 217-227.

Watson, D., Clark, L. A., \& Chmielewski, M. (2008). Structures of personality and their relevant to psychopathology: II. Further articulation of a comprehensive unified trait structure. Journal of Personality, 76, 1485-1522.

Widiger, T. A. (2003). Personality disorder and Axis I psychopathology: the problematic boundary of Axis I and Axis II. Journal of Personality Disorders, 17, 90-108.

Widiger, T. A. (2009). Neuroticism. In M. R. Leary \& R. H. Hoyle (Eds.), Handbook of individual differences in social behavior (pp. 129-146). NY: Guilford.

Widiger, T. A., \& Simonsen, E. (2005). Alternative dimensional models of personality disorder: finding a common ground. Journal of Personality Disorders, 19, 110-130.

Widiger, T. A., \& Trull, T. J. (2007). Plate tectonics in the classification of personality disorder: shifting to a dimensional model. American Psychologist, 62, 71-83.

Widiger, T. A., \& Smith, G. T. (2008). Personality and psychopathology. In O. P. John, R. Robins, \& L. A. Pervin (Eds.), Handbook of personality: Theory and research (3rd ed., pp. 743-769). NY: Guilford.

Widiger, T. A., Frances, A., Spitzer, R., \& Williams, J. (1988). The DSM-III-R personality disorders: an overview. American Journal of Psychiatry, 145, 786-795.

Wiggins, J. S., \& Pincus, H. A. (1989). Conceptions of personality disorder and dimensions of personality. Psychological Assessment, 1, 305-316.

World Health Organization. (1992). The ICD-10 classification of mental and behavioural disorders. Clinical descriptions and diagnostic guidelines. Geneva, Switzerland: Author. 